\title{
Archaea, specific genetic traits, and development of improved bacterial live biotherapeutic products: another face of next-generation probiotics
}

\author{
Khaled Fadhlaoui $^{1} \cdot$ Marie-Edith Arnal $^{1,2} \cdot$ Matthieu Martineau ${ }^{1} \cdot$ Paméla Camponova $^{1} \cdot$ Bernard Ollivier $^{3}$. \\ Paul W. O'Toole ${ }^{4}$. Jean-François Brugère ${ }^{1}$
}

Received: 18 February 2020 / Revised: 25 March 2020 / Accepted: 31 March 2020

(C) Springer-Verlag GmbH Germany, part of Springer Nature 2020

\begin{abstract}
Trimethylamine (TMA) and its oxide TMAO are important biomolecules involved in disease-associated processes in humans (e.g., trimethylaminuria and cardiovascular diseases). TMAO in plasma (pTMAO) stems from intestinal TMA, which is formed from various components of the diet in a complex interplay between diet, gut microbiota, and the human host. Most approaches to prevent the occurrence of such deleterious molecules focus on actions to interfere with gut microbiota metabolism to limit the synthesis of TMA. Some human gut archaea however use TMA as terminal electron acceptor for producing methane, thus indicating that intestinal TMA does not accumulate in some human subjects. Therefore, a rational alternative approach is to eliminate neo-synthesized intestinal TMA. This can be achieved through bioremediation of TMA by these peculiar methanogenic archaea, either by stimulating or providing them, leading to a novel kind of next-generation probiotics referred to as archaebiotics. Finally, specific components which are involved in this archaeal metabolism could also be used as intestinal TMA sequesters, facilitating TMA excretion along with stool. Referring to a standard pharmacological approach, these TMA traps could be synthesized ex vivo and then delivered into the human gut. Another approach is the engineering of known probiotic strain in order to metabolize TMA, i.e., live engineered biotherapeutic products. These alternatives would require, however, to take into account the necessity of synthesizing the 22 nd amino acid pyrrolysine, i.e., some specificities of the genetics of TMA-consuming archaea. Here, we present an overview of these different strategies and recent advances in the field that will sustain such biotechnological developments.
\end{abstract}

\section{Key points}

- Some autochthonous human archaea can use TMA for their essential metabolism, a methyl-dependent hydrogenotrophic methanogenesis.

- They could therefore be used as next-generation probiotics for preventing some human diseases, especially cardiovascular diseases and trimethylaminuria.

- Their genetic capacities can also be used to design live recombinant biotherapeutic products.

- Encoding of the 22nd amino acid pyrrolysine is necessary for such alternative developments.

Keywords Trimethylamine oxide TMAO - Cardiovascular disease CVD * Archaebiotics · Trimethylamine TMA . Trimethylaminuria TMAU · Live biotherapeutic products LBP

Jean-François Brugère

J-Francois.Brugere@uca.fr

1 CNRS, Laboratoire Microorganismes: Génome et Environnement LMGE, Université Clermont Auvergne,

F-63000 Clermont-Ferrand, France
2 Present address: CEA, CNRS, IRIG, SyMMES, Chimie Interface Biologie pour l'Environnement, la Santé et la Toxicologie (CIBEST), Univ. Grenoble Alpes, F-38000 Grenoble, France

3 Aix Marseille Université, CNRS, Université de Toulon, IRD, Mediterranean Institute of Oceanography (MIO), Marseille, France

4 School of Microbiology and APC Microbiome Ireland, University College Cork, Cork, Ireland 


\section{Introduction}

The human gut microbiota contains about as many cells as human ones themselves (Sender et al. 2016). It is a complex mix of microbes from the three domains of life (Bacteria, Archaea, and Eukarya) which differs between human subjects (Arumugam et al. 2011; Donaldson et al. 2016; Human Microbiome Project 2012). It is considered similar to a human organ (the "forgotten organ") that participates in several important physiological processes in humans, encompassing notably nutrition (digestion of fibers, vitamins, and short-chain fatty acids synthesis) and protection against pathogens, as well as having endocrine functions (Clarke et al. 2014; O'Hara and Shanahan 2006). It has co-evolved with its host and provides genes and functions not encoded by the human host, with microbial genes accounting about 100 times more than the human genome itself (Arumugam et al. 2011; Qin et al. 2010). All of this leads one to consider humans and their microbes as a biological entity, a holobiont (Simon et al. 2019). Bacteria are the most represented microbial part of the gut microbiota, and also the most studied: they account for trillions in each individual and represent about $98 \%$ of all microbes (Zhernakova et al. 2016). Eukaryotic cells are far less abundant with yeasts being predominant among them. In-between, archaea account in mean for $1-2 \%$ of all microbes with high variations among subjects (Zhernakova et al. 2016). While the diversity of these human-hosted archaea is likely still underestimated, they are mainly anaerobes performing methanogenesis (Bang and Schmitz 2018; Gaci et al. 2014; Moissl-Eichinger et al. 2018). The main representative of such organisms in the gut is the Methanobacteriales representative Methanobrevibacter smithii (Miller et al. 1982).

Less than a decade ago, a small organic plasma molecule was identified as an important risk factor for cardiovascular disease (CVD), plasma trimethylamine oxide (pTMAO) (Wang et al. 2011). It originates exclusively from the metabolic action of the gut microbiota on some various nutrients leading to one deleterious intestinal intermediary compound further oxidized by the liver. Although the role of pTMAO is a matter of debate as for example high fat-fed mice exposed to TMAO have improved glucose tolerance (Cho and Caudill 2017; Dumas et al. 2017; Jaworska et al. 2019), pTMAO has been shown to be directly or indirectly involved in various mechanisms that drives cardiovascular events, like contribution to the formation of atherosclerotic plaques, foam cells, and platelet activation (Warrier et al. 2015; Zhu et al. 2016) (see (Tang and Hazen 2017; Zeisel and Warrier 2017) for a review). Since, pTMAO has also been linked to various diseases through this "diet-microbe morbid union" (Rak and Rader 2011).Plasma TMAO is, for example, also adversely associated to chronic renal diseases (Tang et al. 2015), nonalcoholic fatty liver disease NAFLD (Chen et al. 2016), type-2 diabetes (Shan et al. 2017; Tang et al. 2017), or colorectal and prostate cancer (Mondul et al. 2015; Xu et al. 2015). Also, it has been recently shown that the gut microbial intermediary compound itself, trimethylamine (TMA) has haemodynamic effects that TMAO does not have, with a significant increased arterial blood pressure (Jaworska et al. 2019). At last, TMA is also involved directly in a rare inherited metabolic disorder named trimethylaminuria (TMAU) or fish odor syndrome in addition to also acquired/transient cases (Christodoulou 2012; Humbert et al. 1970; Messenger et al. 2013). This is a psychosocially distressing disease (see below for reasons and mechanisms).

This peculiar case by which the gut microbiota contributes to disease provides an example on which specific, original, and rational strategies could be applied, which in turn can provide new biotechnological approaches for other (gut microbiota function-derived) diseases.

\section{The gut microbiota and intestinal TMA standing at the crossroads}

At the crossroads between diet and plasma, TMAO/TMA stands the gut microbiota (Fig. 1). The latter generates intestinal TMA, next absorbed by the human host, conveyed into the liver by the portal vein where it is normally oxidized into pTMAO. This oxidation is driven by hepatic flavine monoxygenases and more specifically FMO3 (Bennett et al. 2013; Warrier et al. 2015; Zhu et al. 2018). The elimination of pTMAO will next be the result of renal excretion (Gessner et al. 2018). In some cases, however, impairment of this liver oxidation avoids total or partial conversion of TMA into TMAO, leading to significant levels of TMA in blood which further diffuses in body fluids and is excreted through sweat and breath in addition to urine. The main cause of FMO3 deficiency (i.e., of liver TMA oxidation) relies on mutations in the $f m o 3$ gene, leading to a recessive hereditary metabolic disease, trimethylaminuria (TMAU) (Dolphin et al. 1997). TMA is a volatile molecule which characterizes fish spoilage, resulting from the microbial reduction of TMAO present in high level in seafood for osmoregulation (Ghaly et al. 2010). Humans are very sensitive to this rotten fish (mal)odor, which is interpreted as a protective reflex acquired through evolution, preventing us to eat spoiled food. Consequently, diet does not provide TMA directly because food would be perceived as improper to consume by humans. However, a bad consequence of the distasteful perception of TMA is for patients suffering from TMAU, as they have to handle socially their unpleasant body odor: this often leads to impaired social life, isolation, and depression of patients (Christodoulou 2012; Mackay et al. 2011).

While no TMA is directly consumed, at least four different nutrients are involved in the generation of TMA in the human gut (Fig. 1). First, TMAO itself is such a diet component 


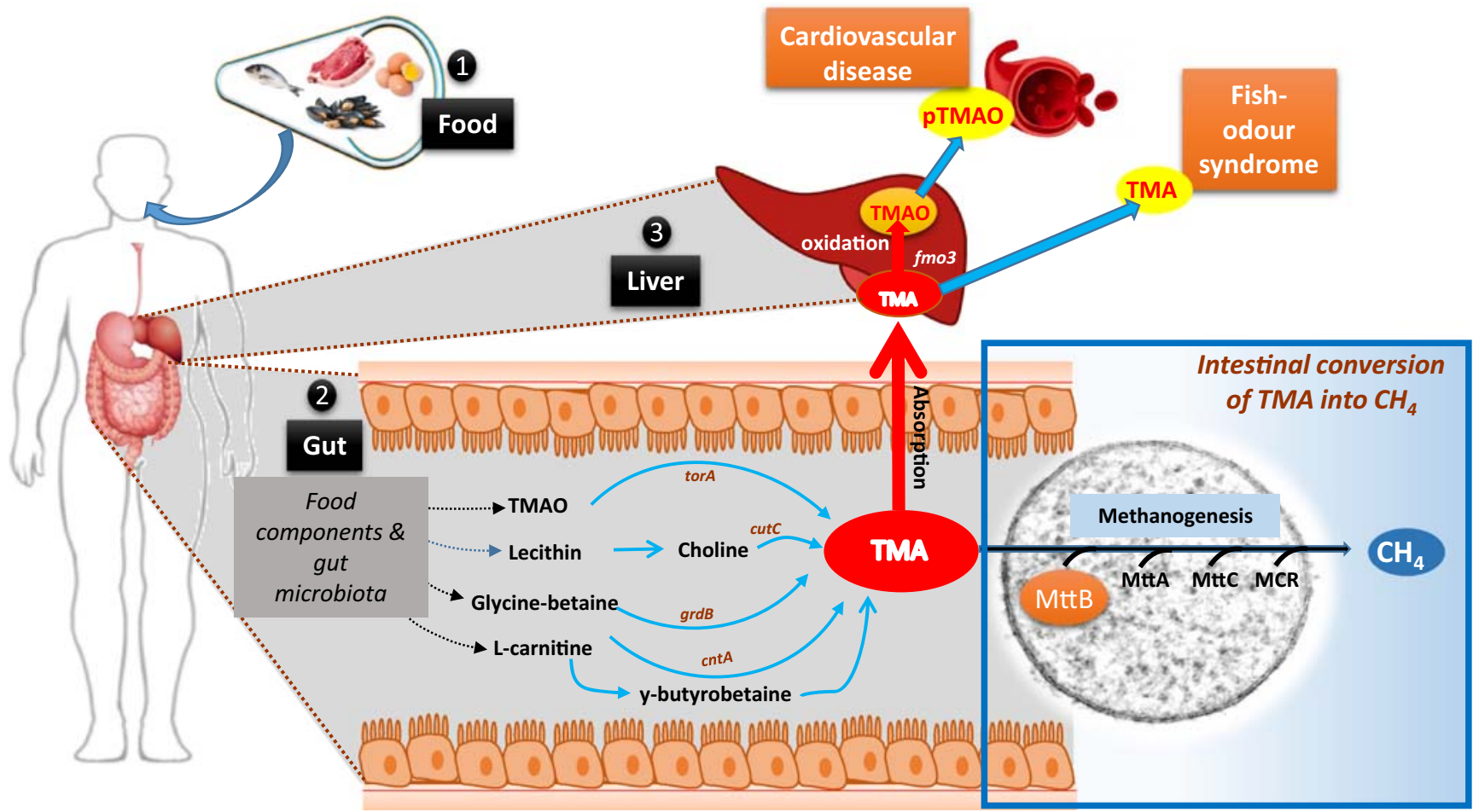

Fig. 1 Origin and fate of intestinal TMA. Intestinal TMA originates from various nutrients from diet (1) which are further metabolized in the gut by bacteria from the microbiota (2). Nutrients are indicated in bold, bacterial genes encoding necessary enzymatic activities in red and italics. TMA is next absorbed and either oxidized into pTMAO in the liver or not, contributing respectively to cardiovascular disease or trimethylaminuria (3).
However, intestinal TMA is not an end-product of the gut microbiota in some humans who host particular methanogenic archaea (down-right), which use TMA $\left(+\mathrm{H}_{2}\right)$ for their methanogenesis, an essential metabolism for their survival. Methyltransferases used for this metabolism are indicated, with an emphasis of the first necessary one, MttB, a pyrrolysinecontaining protein. See text for further details mainly found in seafood. It is an intestinal TMA precursor through reduction by gut bacteria (and next oxidized by the liver back into TMAO), providing an example of metabolic retro conversion in humans (Hoyles et al. 2018). Involved gut bacteria encompass various species with members of Enterobacteriaceae as main contributors (e.g., Escherichia coli and Klebsiella pneumoniae). Other dietary quaternary ammonium compounds that contribute to intestinal TMA are mainly choline-lecithin and L-carnitine (Al-Waiz et al. 1992; Koeth et al. 2013; Wang et al. 2011) and also some others (Jameson et al. 2016). They all require specific enzymatic bacterial activities to be converted into intestinal TMA. Some of these enzymes have only very recently been identified and described (Craciun and Balskus 2012; Martinez-del Campo et al. 2015; Zhu et al. 2014). Also, the potential of each individual to convert these compounds into TMA is highly dependent on their gut microbiota composition, which shows a high inter-individual variation. For example, subjects with vegan diet were shown to not convert (or less) L-carnitine into TMA (Koeth et al. 2013): it is likely that the diet habits of such subjects lead to a counter-selection of L-carnitine metabolizing gut bacteria over time. Moreover, gut bacteria that support TMA synthesis from these various compounds are from various phylogenetic lineages supposedly due to lateral gene transfers having occurred during (gut bacteria) evolution. In consequence, it remains highly speculative to deduce from the gut microbiota composition (i.e., obtained by 16S rRNA gene sequencing) its ability to generate intestinal TMA. This is likely less speculative from metagenomics data from which the presence and proportion of microbial genes involved in TMA synthesis are determined (Borrel et al. 2017; Jameson et al. 2016; Rath et al. 2020).

\section{A strategy designed rationally from the mechanisms: the role of human archaea}

\section{Acting on the gut microbiota metabolism to limit PTMAO}

From above, many potential ways to limit circulating pTMAO and/or intestinal TMA can be imagined. However, most of them have serious disadvantages and side effects. A first idea can be to reduce the amounts of TMA-generating nutrients by selecting foods that provide lower quantities of these different molecules. However, these compounds are present in so many different foods that it makes difficult to address this question, 
especially at the population level. Some foods are however more prone to allow such kind of intervention: for example, eggs are known to contribute a lot for choline-lecithin, red meat for L-carnitine and choline-lecithin, seafood for TMAO and glycine-betaine, and also vegetables and seeds (e.g., quinoa, wheat, or spinach for glycine-betaine). Even more important, these nutrients by themselves, if they are not metabolized into intestinal TMA, are beneficial to humans or even essential. For example, choline is an essential nutrient whose deficiency leads to severe liver dysfunctions with steatosis and neurological disorders (Ueland 2011; Zeisel 1990). At the opposite side, we could imagine the development and use of drugs inhibiting pTMAO synthesis in the liver: this could be achieved by $\mathrm{FMO}_{3}$ inhibitors to avoid oxidation of gut-generated TMA. TMAU patients would not benefit from such a drug, and this would also generate a transient fish odor syndrome as a side effect for others. Therefore, acting on the gut microbiota and on its metabolism itself seems the lonely reliable mean, and for which various complementary ways are possible. This encompasses notably prebiotics and probiotics, but also drugs or food components that would have a (selective) inhibitory action on bacterial enzymes involved in gut TMA synthesis. Among possible drugs, 3,3-dimethyl-1-butanol or DMB shows interesting inhibitory properties of bacterial TMA lyases (Wang et al. 2015). This approach allows now a rational screening of possible inhibitors as recently illustrated by S. Hazen and collaborators (Roberts et al. 2018). Together with the development of drugs targeting specifically some gut microbiota functions, a recent review in this journal described microbes and probiotics associated to decreased pTMAO (Din et al. 2019). However, this review overlooked another, microbiome-based way to limit pTMAO, namely the usage of a unique metabolic property of some human-associated archaea.

\section{Naturally occurring human methanoarchaea use TMA}

Human archaea colonize any human inner or outer surface, as bacteria do even if it is most often in a lesser extent (Pausan et al. 2019). They are of various genera depending on the human biogeographic area, remain likely underestimated, as well considering their amount, diversity, metabolisms, functions, and contributions in each ecosystem (Bang and Schmitz 2018; Gaci et al. 2014; Koskinen et al. 2017). In the gut, these archaea are mainly strict anaerobes producing methane with the property to use $\mathrm{H}_{2}$ as electron donor, i.e., being all hydrogenotrophs. They thus act on one major end-product of the bacterial gut fermentations, $\mathrm{H}_{2}$, and therefore allow to keep low pressure of $\mathrm{H}_{2}$ and in consequence, improve fermentations yields performed by fermentative bacteria that would otherwise been inhibited. According to some large-scale metagenomics studies, they represent in mean $1-2 \%$ of the gut microbes (Zhernakova et al. 2016) but being highly variable in amount among subjects. In the human gut, the main representative is Methanobrevibacter smithii, a Methanobacteriales-affiliated species with a high prevalence (considered to be hosted by more than $50 \%$ of people with variations according to the considered population): it generates methane using $\mathrm{H}_{2}$ as electron donor and $\mathrm{CO}_{2}$ as electron acceptor.

Considering the aim of this article, there are two interesting features of human gut methanogens. First, none has been shown to be pathogenic, which is also the feature of any archaea towards plants or any animal (Cavicchioli et al. 2003; Chaudhary et al. 2018; Gaci et al. 2014; Moissl-Eichinger et al. 2018). In humans, some have been associated to pathologies (e.g., periodontis) but with no evidence of a direct role on them (Horz et al. 2015; Lepp et al. 2004; Sogodogo et al. 2019). However, another methanoarchaeon of the order Methanobacteriales, namely Methanosphaera stadtmanae, has strong inflammatory properties, able to activate human monocyte-derived dendritic cells (Bang et al. 2014; Vierbuchen et al. 2017). This is not the case for other human gut methanogens tested so far (Bang et al. 2017). Second, some specific archaea are able to produce methane using $\mathrm{H}_{2}$ as electron donor and TMA as terminal electron acceptor. This unique metabolism was initially deduced from genomic insights of a human archaeon, Candidatus (Ca.) Methanomethylophilus alvus Mx1201 (Borrel et al. 2012), known to be a representative of a new, 7th methanogenic archaeal taxonomic order (Borrel et al. 2013b; Mihajlovski et al. 2008). Interestingly, a first isolate of this new order named Methanomassiliicoccales was obtained from a human stool sample, Methanomassiliicoccus luminyensis (Dridi et al. 2012; Iino et al. 2013). This microorganism was demonstrated to convert in vitro TMA into methane using $\mathrm{H}_{2}$ according to the metabolic pathway schematically shown in Fig. 1. This was also confirmed using culture enrichments of $\mathrm{Ca}$. M. alvus (Borrel et al. 2017; Brugere et al. 2014) as well with a first isolate of this species, Ca. Methanomethylophilus alvus strain Mx-05 (W. Ben Hania, K. Fadhlaoui et al., in prep.).

\section{Archaea, potential but neglected next-generation probiotics}

\section{Distinctive features of Methanomassiliicoccales}

Methanogenesis is the essential and the only energyyielding metabolic pathway for any methanoarchaeon, functionally similar to the cellular respiration in other anaerobes (i.e., an electron transport coupled to translocations of protons, with next an ATP synthase using this proton (or $\mathrm{Na}^{+}$) motive force to generate ATP). Methanomassiliicoccales have several interesting features that are not found in other methanogens (Borrel et al. 
2013b; Borrel et al. 2014; Sollinger et al. 2016), encompassing an unusual methanogenesis and energy conservation process coupled to it (Borrel et al. 2014; Kroninger et al. 2016; Kröninger et al. 2019). Most of hydrogenotrophic methanogens use $\mathrm{CO}_{2}$ as electron acceptor leading to the terminal product $\mathrm{CH}_{4}$ by the pathway named hydrogenotrophic methanogenesis (Ferry 2010; Thauer et al. 2008). There are a few exceptions regarding hydrogenotrophs unable to reduce $\mathrm{CO}_{2}$ into methane, the main being Methanosphaera spp. (belonging to the order Methanobacteriales as does M. smithii), which can oxidize hydrogen using methanol as terminal electron acceptor. All members of Methanomassiliicoccales however are exceptions, not having the hydrogenotrophic methanogenesis pathway, therefore being unable to perform methanogenesis from $\mathrm{CO}_{2}$ : they all use $\mathrm{C} 1$ compounds as electron acceptors, with some distinctions among them. So far, all of them can use methanol, while some of them can also use monomethylamine (MMA) (Lang et al. 2015), and among them, some can also use TMA (and dimethylamine DMA). In fact, such a metabolism on methylamines relies on a rare and original genetic trait (see chapter 5.1). Some other methanogens perform other kind of methanogenesis, either using $\mathrm{C} 1$ compounds as electron donors instead of $\mathrm{H}_{2}$ (methylotrophs, performing a methylotrophic methanogenesis), or acetate (aceticlastic methanogens) (Costa and Leigh 2014; Garcia et al. 2000; Lyu et al. 2018).

Another unique feature among Methanomassiliicoccales is their phylogenetic situation split into two main clades reflecting roughly an ecological distribution. One clade, to which Ca. M. alvus belongs, is exclusively formed of several candidate species and genera that are to be found in digestive tracts of animals, encompassing insects, poultry, pigs, ruminants, and humans (Borrel et al. 2013b; Mihajlovski et al. 2008; Mihajlovski et al. 2010; Paul et al. 2012; Soellinger et al. 2016). It forms the host-associated clade or HAc (Borrel et al. 2017; Soellinger et al. 2016). The other clade is formed of members found in environments and not in or on an host, with however at least one interesting exception (see below, Ca. Methanomassiliicoccus intestinalis $\mathrm{Mx} 01$ ): all other are freely found in various anaerobic environments like seafloors, freshwater sediments, wetlands, and peatlands for example (Borrel et al. 2017; Borrel et al. 2013b; Soellinger et al. 2016). It forms the free-living clade FLc to which Methanomassiliicoccus luminyensis B10 belongs. The latter was, however, isolated from human stools (Dridi et al. 2012), so that it remains unclear if the natural habitat of this species is really (human or other animal) digestive environments, or is another one, and so if this species was only transiently present (or exceptionally living) in this human subject. This species has never been clearly demonstrated again to live within the human gut: it has sometimes been found in other studies using $\mathrm{PCR} / \mathrm{qPCR}$ tools in humans, which is likely the result of a cross-signal with another Methanomassiliicoccales from this clade, Candidatus Methanomassiliicoccus intestinalis, also named Mx-01 (Borrel et al. 2013a): different strains of Mx01 , phylogenetically closely related to $M$. luminyensis have been identified in various studies and several subjects (e.g., (Borrel et al. 2017; Mihajlovski et al. 2010)), forming one notable exception among FLc members. Therefore, it is assumed that $M$. luminyensis is not a typical representative of the gut-associated Methanomassiliicoccales, which could affect its efficacy to thrive in the human gut.

\section{Using these natural properties to design probiotics from the archaeal domain: archaebiotics}

If we sum up what is mentioned above, TMA is a deleterious molecule synthesized by gut microbes originally believed to be an end-product of the diet-derived metabolism of the gut microbiota. It seems not to be the case in subjects who house some peculiar archaea belonging to the order Methanomassiliicoccales, which have the property to convert TMA into methane (see below for arguments). Methane can be considered as an inert gas for the host, especially at the amount that would be derived from TMA. These archaea are well adapted to this environment, at least for members from the host-associated clade. Moreover, they are very likely safe: this can be first presumed from our knowledge about the archaeal world (no pathogens described so far in humans, animals or plants). Also, M. luminyensis, which very likely does not usually thrive in humans, does not lead in vitro to activation of human monocyte-derived dendritic cells (Bang et al. 2017), a difference with another human-hosted archaeon, Methanosphaera stadtmanae (Bang et al. 2014; Vierbuchen et al. 2017). Based on this, it is logical to propose the use of such archaea as live biotherapeutic agents (Brugere et al. 2014), referred as "Archaebiotics," or probiotics from the domain Archaea. Several data described below support the functionality of such an approach.

In vivo in the human gut, TMA is very likely used by Methanomassiliicoccales which have the genetic potential to use it. In fact, it may be suggested that TMA use is restricted to these microorganisms, conferring them an ecological niche in the human digestive tract: from the Eldermet cohort, an Irish cohort of elderly people (Claesson et al. 2011; Claesson et al. 2012), it has been shown that the TMA level in stools was significantly higher in people in whom no Methanomassiliicoccales were detected, or who had Methanomassiliicoccales (genetically) not able to use trimethylamines, as determined by qPCR and metagenomics data of more than 100 subjects (Borrel et al. 2017) (Fig. 2a). Moreover, the mean level of intestinal TMA was lower when subjects were hosting more than $10^{8}$ TMA-using 


\section{a Humans}

$>10^{8}$ Methanomassiliicoccales (encoding MttB)

$<10^{8}$ Methanomassiliicoccales (encoding MttB)

No Methanomassiliicoccales

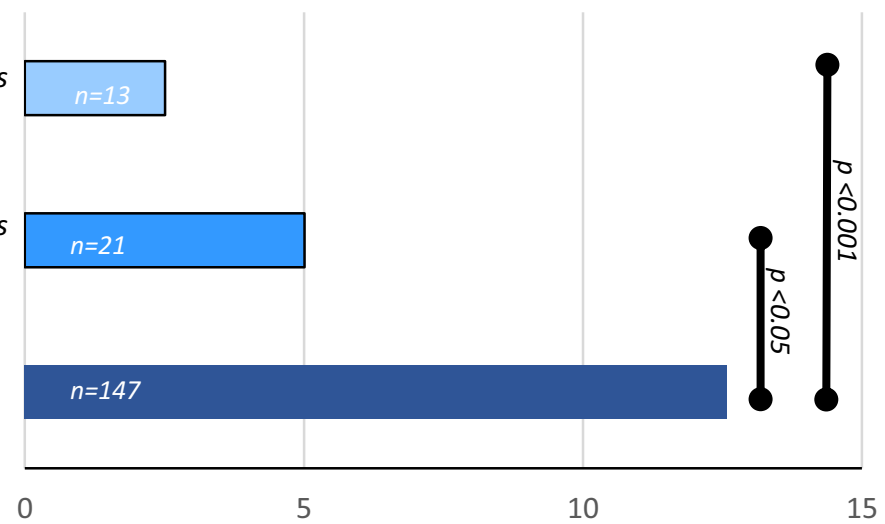

Fecal TMA concentration, $\mu \mathrm{M}$

\section{b Mice}

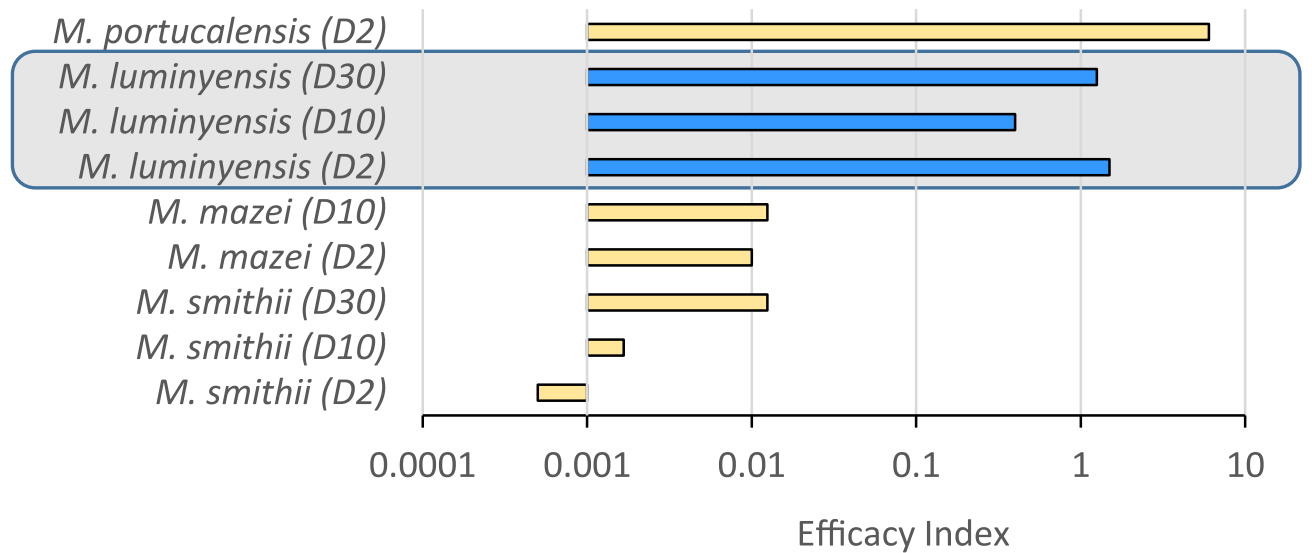

Fig. 2 Influence of Methanomassiliicoccales on fecal TMA and plasma TMAO. a In the subjects from the ELDERMET cohort, a significantly lower fecal TMA level for subjects hosting MttB-encoding Methanomassiliicoccales was observed, more pronounced for subjects hosting higher amounts of these archaea (derived from Fig. 3d in Borrel et al., 2017). Only mean values and statistical significance are indicated. b Archaebiotics in mice: Raj et collaborators reported the beneficial effect of some archaea on plasma TMAO levels in mice (Ramezani et al. 2018): after antibiotics treatment followed by a unique gavage with some different methanogens, pTMAO was assayed at different time points (days 2,

Methanomassiliicoccales (based on a molecular metabolic marker) than less than $10^{8}$, corresponding also with a higher significance of this difference in intestinal TMA level with subjects not hosting Methanomassiliicoccales or not TMAusing Methanomassiliicoccales. Unfortunately, no data are available from this study to know if this lower level of TMA in stools is also associated with lower TMAO level in plasma.

The archaebiotics concept was recently tested on mice (Ramezani et al. 2018). In preliminary experiments, laboratory mice were treated with antibiotics to favor persistence/ implantation of archaea, and fed with a choline-enriched diet. Tested archaea were various mesophilic methanogens provided by a unique gavage of about $10^{8}$ cells after antibiotics
10 , and 30 after inoculation, respectively indicated as D2, D10, and D30). Some archaea and time points (reported at left) gave significant differences compared to pTMAO in control mice (which are reported here). From the results given in the original work (text + figures), an efficacy index for each species of methanogens is calculated to take into account the amount of archaea which were implemented: this index corresponds to the fold of reduction of pTMAO (pTMAO ratios between control and tests) divided by the amount of archaea per $\mu \mathrm{g}$ of stools. The Methanomassiliicoccales $M$. luminyensis, which has a significant effect at the 3 time points, is highlighted (gray box)

treatments. With the exception of Methanobrevibacter smithii (a human-hosted archaeon), they were all known to use TMA for their methanogenesis. Also, they were all known to not thrive in the human (and mouse) gut, with possibly the exception of Methanomassiliicoccus luminyensis which belongs to the free-living clade (see above). This species was the unique axenic cultivated representative from Methanomassiliicoccales available for experimentation by authors. Surprisingly, gavage with the human archaeon $M$. smithii resulted in significant lower pTMAO levels all along the experiment (days 2, 10, and 30 after the unique gavage) compared to controls. It also improved the health status of $\mathrm{ApoE}^{-/-}$mice compared to controls, i.e., less aortic/atherosclerotic lesions. As it is an archaeon that does not 
use TMA, the beneficial effects of $M$. smithii can be attributed to a modification/modulation of the gut microbiota metabolic activity, with an observed change in its bacterial composition compared to controls (Ramezani et al. 2018). M. smithii was also shown to colonize the mouse gut, at a higher level compared to any other tested archaea. Other (TMA-using) archaea gave significantly lower pTMAO levels, but this effect was detectable only for the first days of experiments after the unique gavage: in fact, these archaea were not retrieved (or at very low levels) at day 30, indicating no colonization for most of them and a continuous disappearance all along the experiment. One exception was however Methanomassiliicoccus luminyensis, which was retrieved in stools at low level from the beginning to the end of the experiment. If we analyze more deeply data provided by Ramezani et coll. (Ramezani et al. 2018) and using numerical approximations from the figures and text, we can compare the effectiveness of each archaeal species which lowered significantly pTMAO taking into account the count of archaea retrieved in mice stools at each time points (Fig. 2b). M. smithii has in fact a significant effect of pTMAO level associated to a high count of $M$. smithii in stools. This corresponds to a lower reduction of pTMAO per cell of M. smithii varying across time points: this argues for an indirect action of $M$. smithii on intestinal TMA/pTMAO, likely by acting on the gut microbial composition. Interestingly, the use of the Methanomassiliicoccales representative M. luminyensis gave a significant reduction of pTMAO all over the 30 days of experiments. It had however a low count in stools, but one of the highest and quite similar efficacy per cell at the three time points (see Box in Fig. 2b). This indicates that M. luminyensis has a strong effect that can be detected at even low colonization level, likely by acting directly on intestinal TMA through a conversion into methane. These results are in fact in line with observations made in humans of the Eldermet cohort (Borrel et al. 2017). Moreover, the low level of M. luminyensis observed in mice stools may be the result of a methanogen not well adapted to thrive in the gut, especially in mice: the isolation of Ca. Methanomethylophilus alvus (W. Ben Hania, K. Fadhlaoui et al., in prep.) gives now the opportunity to carry out similar experiments with a human-hosted Methanomassiliicoccales from the host-associated clade, which should indicate more precisely the interest (or not) to follow up a development and use of archaebiotics.

\section{Future developments: genetics of archaea as a tool for next-generation probiotics}

It is shown from above that only archaea, and among them, only some methanoarchaea are currently known to have properties to remediate TMA in the gut, with a few of them naturally occurring in human gut. It is however imaginable that some of these metabolic properties could be used to provide alternative solutions in human health. Among these solutions, the specificity of enzymes used in this methylotrophic metabolism could be used to sequester TMA in the human gut, in a similar but more specific way than charcoal does, this last being sometimes used for TMAU patients to lower TMA absorption. The first archaeal enzyme of this pathway, MttB, can be considered for such an application. This is the one that recognizes TMA to further transfer a methyl-group to several other methyltransferases (a corrinoid protein MttC, next another methyltransferase MttA which methylates coenzyme M; see Fig. 1).

\section{Pyrrolysine, a necessary unusual amino acid in anaerobic TMA metabolism}

MttB is a (dimeric) enzyme which has however a striking feature, from which depends its biological activity. For substrate specificity and functional activity, this protein has to possess pyrrolysine (Pyl) in its active site (Hao et al. 2002), hence losing its specificity (Craciun and Balskus 2012). Pyrrolysine is the 22nd proteinogenic amino acid discovered at the beginning of the twenty-first century (Atkins and Gesteland 2002; Srinivasan et al. 2002). It is found quite exclusively in some archaea in which it is intimately linked to methylamines metabolism, especially the methanogenesis from MMA, DMA, and TMA (Brugère et al. 2018). Its synthesis, encoding, phylogenetic origin, and biological properties raise many questions considering archaea and more specifically Methanomassiliicoccales (Brugère et al. 2018). This peculiar amino acid and the system which allows its encoding have led to new biotechnological applications, especially in synthetic biology and for quite any kind of cells (for a recent review, see (Tharp et al. 2018)). In short, this unusual proteinogenic 22nd amino acid is synthesized from one usual amino acid, lysine, which is used twice through 3 reactions catalyzed by 3 different enzymes (PylB, PylC, and PylD) (Fig. 3) (Krzycki 2004; Krzycki 2005). This amino acid is next encoded by the usual way at ribosomes, using a specific tRNA whose anticodon is complementary to the UAG amber codon (usually a stop codon). This tRNA acts as an UAG stop codon suppressor: The mechanisms underlying the insertion in nascent proteins (rather than a stall in-between the mRNA and the ribosome) is still unclear but is likely the result of a competition between archaeal releasing factor aRF and PyltRNA at the ribosome in archaea (Zhang et al. 2005). As mentioned above, Pyl is a necessary component of an active $\mathrm{MttB}$ protein, so that a biotechnological production of $\mathrm{MttB}$ has to rely to organisms or artificial/bioengineered systems/ cells that can encode (and synthesize) Pyl. 


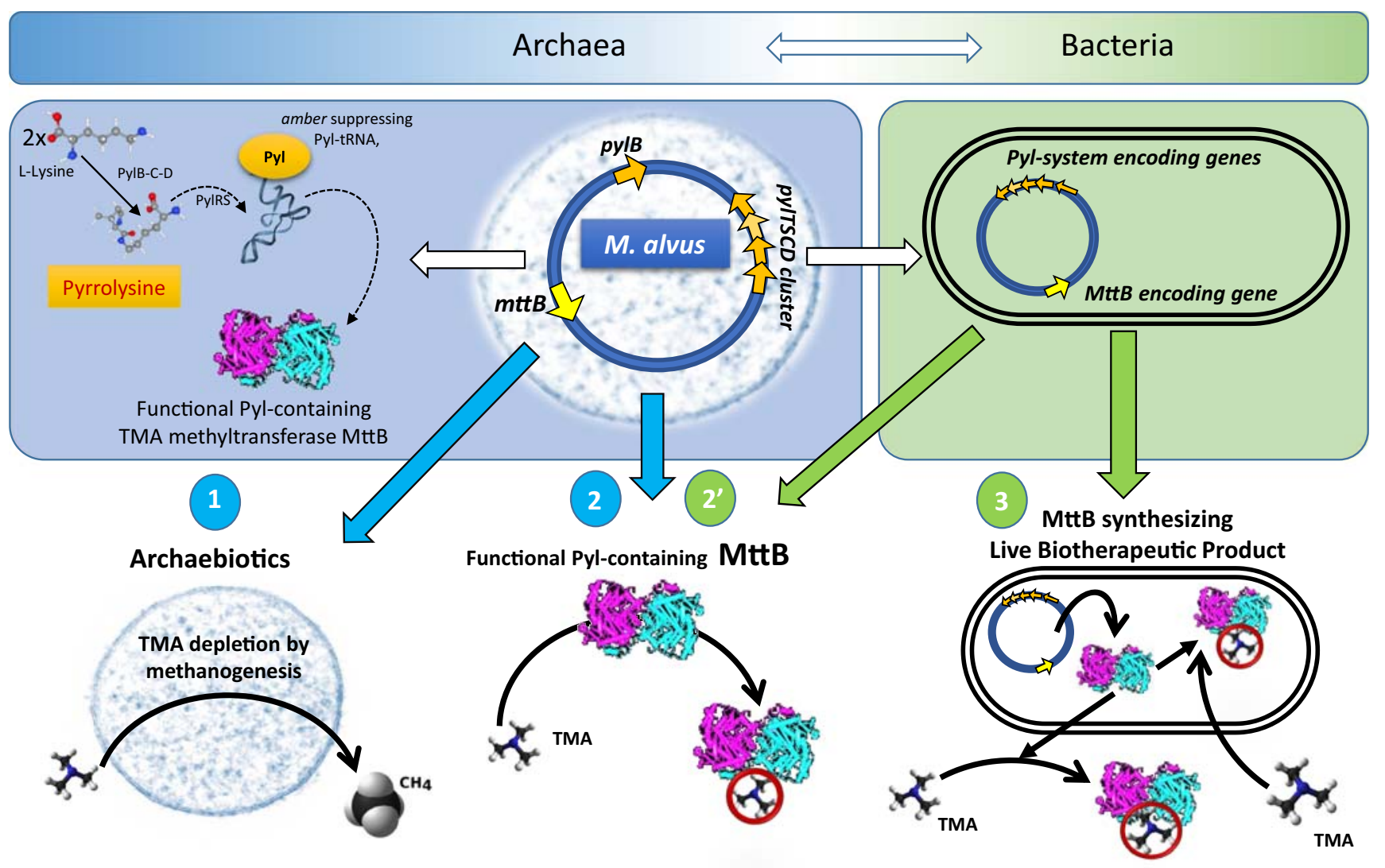

Fig. 3 Biotechnological developments using archaea and genetics of archaea in order to lower intestinal TMA and prevent TMAU and CVD. The Methanomassiliicoccales archaeon Candidatus Methanomethylophilus alvus is represented in the up center of the figure. It is naturally able to synthesize and encode Pyl, the 22nd amino acid through an UAG (amber) suppressing tRNA, which allows the synthesis of a functional methyltransferase specific to TMA (MttB, see also Fig. 1). Therefore, this organism (or any other MttB-encoding archaeon) can be used as a live biotherapeutic archaeon (1) which will convert TMA into methane through its methanogenesis (Archaebiotics). Alternatively, this kind of archaeon can also be used to extract a

\section{Producing and using components from archaea for a pharmaceutical approach}

We therefore propose to engineer microbial cells factories that would be exploited for the controlled production of $\mathrm{MttB}$, next used to be delivered in the human gut to sequester TMA before its absorption like charcoal does unspecifically (Fig. 3). It has been shown for a long time that, for example, E. coli could be transformed by the Pyl genetic components (Longstaff et al. 2007; Namy et al. 2007). For this purpose, MtmB, a Pylprotein which recognizes MMA was synthesized by $E$. coli as a reporter of the functionality of the Pyl-system in this nonnaturally Pyl-encoding bacterium. Recently also, Pyl-tRNA/ Pyl-tRNA synthetase pair from Ca. Methanomethylophilus alvus was shown to have even better yields for site-directed insertion of functional MttB protein that can be further used as a pharmaceutical delivered in the human gut in order to catch TMA (2). Such a protein production and purification can be achieved through the engineering of bacterial cells (or yeasts) factories $\left(2^{\prime}\right)$ with the need of the correct synthesizing and encoding of Pyl. The production of Pyl-containing methyltransferases has already been achieved in E. coli. At last, and alternatively, such an engineered bacterial cell can originate from a known probiotics. This next-generation probiotics can next be used as a live recombinant biotherapeutic product able to synthesize and deliver MttB in situ in the gut to sequester TMA (3), accordingly to what has been already developped for E. coli Nissle 1917 (Isabella et al. 2018)

non-natural amino acids in proteins (Beránek et al. 2019; Willis and Chin 2018). We successfully produced MtmB with the Pylsystem of Ca. M. alvus (unpublished data) and are now engineering various $E$. coli recombinant bacterial cells to produce and/or secrete MttB. This cell or medium/purified protein will be next used in vitro to determine its affinity towards TMA with the aim to sequester TMA in the human gut: properly delivered in the gut, it would link neo-synthesized TMA before its absorption by the gut epithelium and its diffusion to the liver, so either lowering pTMAO (which would prevent cardiovascular disease) or lowering TMA (which would lowered symptoms for TMAU subjects).

Therefore, the genetics of these specific TMAmetabolizing archaea could be used to engineer MttB factoring cells, as producers of a protein drug. 


\section{Upgrading bacterial probiotics with archaeal components: live bioengineered biotherapeutic agents}

While the in vitro synthesis of MttB as a drug is a feasible option, leading in fact to quite a standard pharmaceutical approach, the functionality of such a system in human remains to be tested: especially, the delivery of MttB, whose integrity has to be kept long enough to fix TMA, is a determining step. Another option can be the in situ synthesis of TMA in the human gut, by providing factory cells rather than the protein itself. Live recombinant biotherapeutic agents have been recently shown to be a functional option using a standard probiotic cell that was bioengineered to synthesize and deliver active enzymes in the gut (Kurtz et al. 2018). As an example, the probiotic strain E. coli Nissle 1917 was recently engineered to express specific proteins in gut of mice and monkeys in response to anoxic environments: it allows the expression of enzymes able to convert phenylalanine (Phe) into trans-cinnamate (further converted into hypurate by the host), therefore depleting (diet-derived) Phe (Isabella et al. 2018). This is of particular interest in phenylketonuria, a genetic disease in which unmetabolized Phe can lead to neurotoxicity. This case has many similarity to the one described for intestinal TMA and cardiovascular disease or TMAU. It is now known that $E$. coli survives to the expression of an UAG non-sense codon suppressing tRNA and that the archaeal Pyl-system is functional in this organism. Therefore, it can be proposed first to engineer the probiotic strain E. coli Nissle 1917 to synthesize and encode Pyl, as well as to synthesize (a functionnal) MttB. Other probiotic strains can also to be considered (Fig. 3): the live bioengineered strain, e.g., a recombinant $E$. coli Nissle 1917 would be given as live biotherapeutic product, which would synthesize an active TMA sequestering protein when reaching the gut.

\section{Concluding remarks}

So far, microorganisms belonging to the domain Archaea remain neglected candidates as potential probiotics: no archaeon has been used as a live biotherapeutic product in humans or in any other animal so far, while archaea have specific features that are of great interest for the prevention of important human diseases including cardiovascular diseases and trimethylaminuria. We already know that these properties apparently contribute to limit deleterious effects consecutive to TMA synthesis by bacteria in the gut (Borrel et al. 2017). If not used directly for their properties as live biotherapeutic product (i.e., Archaebiotics), archaea provide unique features that could be engineered in other microbes to provide either pharmaceuticals or live biotherapeutic products. Moreover, it has to be highlighted that so far, no archaeon has been reported as being pathogenic for any plant or animal, and interestingly for humans. This gives value to the use of them for therapeutic purposes, especially as some are natural inhabitants of most host-associated microbiomes. Archaea-based research for alternative solutions in human, animal, or plant health is just reaching its infancy, providing promising new strategies for prophylactics and therapeutics of some important diseases.

\section{Acknowledgements}

JFB acknowledges Dominic S. Raj from The George Washington University School of Medicine, Washington, USA for communications about mice experiments with archaea (Fig. 2b).

Authors' contributions JFB and PWOT are inventors on patent WO2014082773A1 co-owned by their respective universities. PWOT is a founder of 4D Pharma Cork Ltd., a role that has not constrained or influenced this review. All the authors contributed to the written of the manuscript and the conception and design of figures. All the authors read and approved the manuscript.

Funding information JFB is supported by a grant from the Agence Nationale de la Recherche of the French government through the program "Investissements d'Avenir" (16-IDEX-0001 CAP 20-25), through an "Innovation-Research" grant from Hub Innovergne. Work in PWOT's lab is supported by an award from Science Foundation Ireland to APC Microbiome Ireland.

\section{Compliance with ethical standards}

Conflict of interests The authors declare that they have no conflict of interest.

Ethical approval This article does not contain any studies with human participants or animals performed by any of the authors.

\section{References}

Al-Waiz M, Mikov M, Mitchell S, Smith R (1992) The exogenous origin of trimethylamine in the mouse. Metabol 41(2):135-136

Arumugam M, Raes J, Pelletier E, Le Paslier D, Yamada T, Mende DR, Fernandes GR, Tap J, Bruls T, Batto JM, Bertalan M, Borruel N, Casellas F, Fernandez L, Gautier L, Hansen T, Hattori M, Hayashi T, Kleerebezem M, Kurokawa K, Leclerc M, Levenez F, Manichanh C, Nielsen HB, Nielsen T, Pons N, Poulain J, Qin J, Sicheritz-Ponten T, Tims S, Torrents D, Ugarte E, Zoetendal EG, Wang J, Guarner F, Pedersen O, de Vos WM, Brunak S, Dore J, Antolin M, Artiguenave F, Blottiere HM, Almeida M, Brechot C, Cara C, Chervaux C, Cultrone A, Delorme C, Denariaz G, Dervyn R, Foerstner KU, Friss C, van de Guchte M, Guedon E, Haimet F, Huber W, van Hylckama-Vlieg J, Jamet A, Juste C, Kaci G, Knol J, Lakhdari O, Layec S, Le Roux K, Maguin E, Merieux A, Melo Minardi R, M'Rini C, Muller J, Oozeer R, Parkhill J, Renault P, Rescigno M, Sanchez N, Sunagawa S, Torrejon A, Turner K, Vandemeulebrouck G, Varela E, Winogradsky Y, Zeller G, Weissenbach J, Ehrlich SD, 
Bork P (2011) Enterotypes of the human gut microbiome. Nature 473(7346):174-180. https://doi.org/10.1038/nature09944

Atkins JF, Gesteland R (2002) Biochemistry. The 22nd amino acid. Science (New York, NY) 296(5572):1409-1410. https://doi.org/ 10.1126/science. 1073339

Bang C, Schmitz RA (2018) Archaea: forgotten players in the microbiome. Emerg Top Life Sci 2(4):459-468

Bang C, Vierbuchen T, Gutsmann T, Heine H, Schmitz RA (2017) Immunogenic properties of the human gut-associated archaeon Methanomassiliicoccus luminyensis and its susceptibility to antimicrobial peptides. PLoS One 12(10):e0185919-e0185919. https:// doi.org/10.1371/journal.pone.0185919

Bang C, Weidenbach K, Gutsmann T, Heine H, Schmitz RA (2014) The intestinal archaea Methanosphaera stadtmanae and Methanobrevibacter smithii activate human dendritic cells. PLoS One 9(6):e99411

Bennett BJ, de Aguiar Vallim TQ, Wang Z, Shih DM, Meng Y, Gregory J, Allayee H, Lee R, Graham M, Crooke R (2013) Trimethylamine-Noxide, a metabolite associated with atherosclerosis, exhibits complex genetic and dietary regulation. Cell Metab 17(1):49-60

Beránek V, Willis JCW, Chin JW (2019) An evolved Methanomethylophilus alvus pyrrolysyl-tRNA synthetase/tRNA pair is highly active and orthogonal in mammalian cells. Biochem 58(5):387-390. https://doi.org/10.1021/acs.biochem.8b00808

Borrel G, Harris HM, Parisot N, Gaci N, Tottey W, Mihajlovski A, Deane J, Gribaldo S, Bardot O, Peyretaillade E, Peyret P, O'Toole PW, Brugere JF (2013a) Genome sequence of "Candidatus Methanomassiliicoccus intestinalis" issoire-Mx1, a third Thermoplasmatales-related methanogenic archaeon from human feces. Genome Announc 1(4). https://doi.org/10.1128/genomeA. 00453-13

Borrel G, Harris HM, Tottey W, Mihajlovski A, Parisot N, Peyretaillade E, Peyret P, Gribaldo S, O'Toole PW, Brugere JF (2012) Genome sequence of "Candidatus Methanomethylophilus alvus" Mx1201, a methanogenic archaeon from the human gut belonging to a seventh order of methanogens. J Bacteriol 194(24):6944-6945. https://doi. org/10.1128/JB.01867-12

Borrel G, McCann A, Deane J, Neto MC, Lynch DB, Brugere JF, O'Toole PW (2017) Genomics and metagenomics of trimethylamineutilizing Archaea in the human gut microbiome. ISME J 11(9): 2059-2074. https://doi.org/10.1038/ismej.2017.72

Borrel G, O'Toole PW, Harris HM, Peyret P, Brugere JF, Gribaldo S (2013b) Phylogenomic data support a seventh order of methylotrophic methanogens and provide insights into the evolution of methanogenesis. Genome Biol Evol 5(10):1769-1780. https:// doi.org/10.1093/gbe/evt128

Borrel G, Parisot N, Harris HM, Peyretaillade E, Gaci N, Tottey W, Bardot O, Raymann K, Gribaldo S, Peyret P, O'Toole PW, Brugere JF (2014) Comparative genomics highlights the unique biology of Methanomassiliicoccales, a Thermoplasmatales-related seventh order of methanogenic archaea that encodes pyrrolysine. BMC Genomics 15:679. https://doi.org/10.1186/1471-2164-15-679

Brugère J-F, Atkins JF, O'Toole PW, Borrel G (2018) Pyrrolysine in archaea: a 22nd amino acid encoded through a genetic code expansion. Emerg Top Life Sci 2(4):607-618. https://doi.org/10.1042/ etls20180094

Brugere JF, Borrel G, Gaci N, Tottey W, O'Toole PW, Malpuech-Brugere C (2014) Archaebiotics: proposed therapeutic use of archaea to prevent trimethylaminuria and cardiovascular disease. Gut Microbes 5(1):5-10. https://doi.org/10.4161/gmic.26749

Cavicchioli R, Curmi PMG, Saunders N, Thomas T (2003) Pathogenic archaea: do they exist? Bioessays 25(11):1119-1128. https://doi.org/ 10.1002/bies. 10354

Chaudhary PP, Conway PL, Schlundt J (2018) Methanogens in humans: potentially beneficial or harmful for health. Appl Microbiol
Biotechnol 102(7):3095-3104. https://doi.org/10.1007/s00253018-8871-2

Chen Y-m, Liu Y, Zhou R-f, Chen X-1, Wang C, Tan X-y, Wang L-j, Zheng R-d, Zhang H-w, Ling W-h (2016) Associations of gutflora-dependent metabolite trimethylamine-N-oxide, betaine and choline with non-alcoholic fatty liver disease in adults. Sci Rep 6(1):1-9

Cho CE, Caudill MA (2017) Trimethylamine-N-oxide: friend, foe, or simply caught in the cross-fire? Trends Endocrinol Metab 28(2): $121-130$

Christodoulou J (2012) Trimethylaminuria: an under-recognised and socially debilitating metabolic disorder. J Paediatr Child H 48(3): E153-E155

Claesson MJ, Cusack S, O'Sullivan O, Greene-Diniz R, de Weerd H, Flannery E, Marchesi JR, Falush D, Dinan T, Fitzgerald G, Stanton C, van Sinderen D, O'Connor M, Harnedy N, O'Connor K, Henry C, O'Mahony D, Fitzgerald AP, Shanahan F, Twomey C, Hill C, Ross RP, O'Toole PW (2011) Composition, variability, and temporal stability of the intestinal microbiota of the elderly. Proc Natl Acad Sci U S A 108(Suppl 1):4586-4591. https://doi.org/10. 1073/pnas. 1000097107

Claesson MJ, Jeffery IB, Conde S, Power SE, O'Connor EM, Cusack S, Harris HM, Coakley M, Lakshminarayanan B, O'Sullivan O, Fitzgerald GF, Deane J, O'Connor M, Harnedy N, O'Connor K, O'Mahony D, van Sinderen D, Wallace M, Brennan L, Stanton C, Marchesi JR, Fitzgerald AP, Shanahan F, Hill C, Ross RP, O'Toole PW (2012) Gut microbiota composition correlates with diet and health in the elderly. Nature 488(7410):178-184. https://doi.org/ 10.1038/nature11319

Clarke G, Stilling RM, Kennedy PJ, Stanton C, Cryan JF, Dinan TG (2014) Minireview: gut microbiota: the neglected endocrine organ. Mol Endocrinol 28(8):1221-1238

Costa KC, Leigh JA (2014) Metabolic versatility in methanogens. Curr Opin Biotechnol 29:70-75

Craciun S, Balskus EP (2012) Microbial conversion of choline to trimethylamine requires a glycyl radical enzyme. Proc Natl Acad Sci U S A 109(52):21307-21312. https://doi.org/10.1073/pnas. 1215689109

Din AU, Hassan A, Zhu Y, Yin T, Gregersen H, Wang G (2019) Amelioration of TMAO through probiotics and its potential role in atherosclerosis. Appl Microbiol Biotechnol 103(23-24):92179228. https://doi.org/10.1007/s00253-019-10142-4

Dolphin CT, Janmohamed A, Smith RL, Shephard EA (1997) Missense mutation in flavin-containing mono-oxygenase 3 gene, FMO3, underlies fish-odour syndrome. Nat Genet 17(4):491-494

Donaldson GP, Lee SM, Mazmanian SK (2016) Gut biogeography of the bacterial microbiota. Nat Rev Microbiol 14(1):20-32

Dridi B, Fardeau ML, Ollivier B, Raoult D, Drancourt M (2012) Methanomassiliicoccus luminyensis gen. nov., sp. nov., a methanogenic archaeon isolated from human faeces. Int J Syst Evol Microbiol 62(Pt 8):1902-1907. https://doi.org/10.1099/ijs.0. 033712-0

Dumas M-E, Rothwell AR, Hoyles L, Aranias T, Chilloux J, Calderari S, Noll EM, Péan N, Boulangé CL, Blancher C (2017) Microbial-host co-metabolites are prodromal markers predicting phenotypic heterogeneity in behavior, obesity, and impaired glucose tolerance. Cell Rep 20(1):136-148

Ferry JG (2010) The chemical biology of methanogenesis. Planet Sp Sci 58(14-15):1775-1783

Gaci N, Borrel G, Tottey W, O'Toole PW, Brugere JF (2014) Archaea and the human gut: new beginning of an old story. World J Gastroenterol 20(43):16062-16078. https://doi.org/10.3748/wjg.v20.i43.16062

Garcia J-L, Patel BK, Ollivier B (2000) Taxonomic, phylogenetic, and ecological diversity of methanogenic Archaea. Anaerobe 6(4):205226 
Gessner A, König J, Fromm MF (2018) Contribution of multidrug and toxin extrusion protein 1 (MATE1) to renal secretion of trimethylamine-N-oxide (TMAO). Sci Rep 8(1):1-10

Ghaly AE, Dave D, Budge S, Brooks M (2010) Fish spoilage mechanisms and preservation techniques. Am J Appl Sci 7(7):859

Hao B, Gong W, Ferguson TK, James CM, Krzycki JA, Chan MK (2002) A new UAG-encoded residue in the structure of a methanogen methyltransferase. Science 296(5572):1462-1466. https://doi.org/ 10.1126/science. 1069556

Horz HP, Robertz N, Vianna ME, Henne K, Conrads G (2015) Relationship between methanogenic archaea and subgingival microbial complexes in human periodontitis. Anaerobe 35(Pt A):10-12. https://doi.org/10.1016/j.anaerobe.2015.02.008

Hoyles L, Jiménez-Pranteda ML, Chilloux J, Brial F, Myridakis A, Aranias T, Magnan C, Gibson GR, Sanderson JD, Nicholson JK (2018) Metabolic retroconversion of trimethylamine N-oxide and the gut microbiota. Microbiome 6(1):73

Human Microbiome Project C (2012) Structure, function and diversity of the healthy human microbiome. Nature 486(7402):207-214. https:// doi.org/10.1038/nature11234

Humbert J, Hammond K, Hathaway W, Marcoux J, O'Brien D (1970) Trimethylaminuria: the fish-odour syndrome. Lancet 296(7676): 770-771

Iino T, Tamaki H, Tamazawa S, Ueno Y, Ohkuma M, Suzuki K, Igarashi Y, Haruta S (2013) Candidatus Methanogranum caenicola: a novel methanogen from the anaerobic digested sludge, and proposal of Methanomassiliicoccaceae fam. nov. and Methanomassiliicoccales ord. nov., for a methanogenic lineage of the class Thermoplasmata. Microbes Environ 28(2):244-250

Isabella VM, Ha BN, Castillo MJ, Lubkowicz DJ, Rowe SE, Millet YA, Anderson CL, Li N, Fisher AB, West KA, Reeder PJ, Momin MM, Bergeron CG, Guilmain SE, Miller PF, Kurtz CB, Falb D (2018) Development of a synthetic live bacterial therapeutic for the human metabolic disease phenylketonuria. Nat Biotechnol 36(9):857-864. https://doi.org/10.1038/nbt.4222

Jameson E, Doxey AC, Airs R, Purdy KJ, Murrell JC, Chen Y (2016) Metagenomic data-mining reveals contrasting microbial populations responsible for trimethylamine formation in human gut and marine ecosystems. Microb Genom 2(9)

Jaworska K, Bielinska K, Gawrys-Kopczynska M, Ufnal M (2019) TMA (trimethylamine), but not its oxide TMAO (trimethylamine-oxide), exerts haemodynamic effects: implications for interpretation of cardiovascular actions of gut microbiome. Cardiovasc Res 115(14): 1948-1949

Koeth RA, Wang Z, Levison BS, Buffa JA, Org E, Sheehy BT, Britt EB, $\mathrm{Fu} \mathrm{X,} \mathrm{Wu} \mathrm{Y,} \mathrm{Li} \mathrm{L} \mathrm{(2013)} \mathrm{Intestinal} \mathrm{microbiota} \mathrm{metabolism} \mathrm{of} \mathrm{L-}$ carnitine, a nutrient in red meat, promotes atherosclerosis. Nat Med 19(5):576

Koskinen K, Pausan MR, Perras AK, Beck M, Bang C, Mora M, Schilhabel A, Schmitz R, Moissl-Eichinger C (2017) First insights into the diverse human Archaeome: specific detection of archaea in the gastrointestinal tract, lung, and nose and on skin. mBio 8(6): e00824-e00817. https://doi.org/10.1128/mBio.00824-17

Kroninger L, Berger S, Welte C, Deppenmeier U (2016) Evidence for the involvement of two heterodisulfide reductases in the energyconserving system of Methanomassiliicoccus luminyensis. FEBS J 283(3):472-483. https://doi.org/10.1111/febs.13594

Kröninger L, Steiniger F, Berger S, Kraus S, Welte CU, Deppenmeier U (2019) Energy conservation in the gut microbe Methanomassiliicoccus luminyensis is based on membrane-bound ferredoxin oxidation coupled to heterodisulfide reduction. FEBS J 286(19):3831-3843

Krzycki JA (2004) Function of genetically encoded pyrrolysine in corrinoid-dependent methylamine methyltransferases. Curr Opin Chem Biol 8(5):484-491. https://doi.org/10.1016/j.cbpa.2004.08. 012
Krzycki JA (2005) The direct genetic encoding of pyrrolysine. Curr Opin Microbiol 8(6):706-712

Kurtz C, Denney WS, Blankstein L, Guilmain SE, Machinani S, Kotula J, Saha S, Miller P, Brennan AM (2018) Translational development of microbiome-based therapeutics: kinetics of E. coli Nissle and engineered strains in humans and nonhuman primates. Clin Transl Sci 11(2):200-207. https://doi.org/10.1111/cts.12528

Lang K, Schuldes J, Klingl A, Poehlein A, Daniel R, Brune A (2015) New mode of energy metabolism in the seventh order of methanogens as revealed by comparative genome analysis of "Candidatus Methanoplasma termitum". Appl Environ Microbiol 81(4):1338-1352

Lepp PW, Brinig MM, Ouverney CC, Palm K, Armitage GC, Relman DA (2004) Methanogenic Archaea and human periodontal disease. Proc Natl Acad Sci U S A 101(16):6176-6181. https://doi.org/10. 1073/pnas.0308766101

Longstaff DG, Larue RC, Faust JE, Mahapatra A, Zhang L, GreenChurch KB, Krzycki JA (2007) A natural genetic code expansion cassette enables transmissible biosynthesis and genetic encoding of pyrrolysine. Proc Natl Acad Sci U S A 104(3):1021-1026. https:// doi.org/10.1073/pnas.0610294104

Lyu Z, Shao N, Akinyemi T, Whitman WB (2018) Methanogenesis. Curr Biol 28(13):R727-R732

Mackay RJ, McEntyre CJ, Henderson C, Lever M, George PM (2011) Trimethylaminuria: causes and diagnosis of a socially distressing condition. Clin Biochem Rev 32(1):33

Martinez-del Campo A, Bodea S, Hamer HA, Marks JA, Haiser HJ, Turnbaugh PJ, Balskus EP (2015) Characterization and detection of a widely distributed gene cluster that predicts anaerobic choline utilization by human gut bacteria. mBio 6(2). https://doi.org/10. 1128/mBio.00042-15

Messenger J, Clark S, Massick S, Bechtel M (2013) A review of trimethylaminuria:(fish odor syndrome). J Clin Aesthet Dermatol 6(11):45

Mihajlovski A, Alric M, Brugere JF (2008) A putative new order of methanogenic Archaea inhabiting the human gut, as revealed by molecular analyses of the mcrA gene. Res Microbiol 159(7-8): 516-521. https://doi.org/10.1016/j.resmic.2008.06.007

Mihajlovski A, Dore J, Levenez F, Alric M, Brugere JF (2010) Molecular evaluation of the human gut methanogenic archaeal microbiota reveals an age-associated increase of the diversity. Environ Microbiol Rep 2(2):272-280. https://doi.org/10.1111/j.1758-2229.2009. 00116.x

Miller TL, Wolin M, de Macario EC, Macario A (1982) Isolation of Methanobrevibacter smithii from human feces. Appl Environ Microbiol 43(1):227-232

Moissl-Eichinger C, Pausan M, Taffner J, Berg G, Bang C, Schmitz RA (2018) Archaea are interactive components of complex microbiomes. Trends Microbiol 26(1):70-85

Mondul AM, Moore SC, Weinstein SJ, Karoly ED, Sampson JN, Albanes D (2015) Metabolomic analysis of prostate cancer risk in a prospective cohort: the alpha-tocopherol, beta-carotene cancer prevention (ATBC) study. Int J Cancer 137(9):2124-2132

Namy O, Zhou Y, Gundllapalli S, Polycarpo CR, Denise A, Rousset J-P, Söll D, Ambrogelly A (2007) Adding pyrrolysine to the Escherichia coli genetic code. FEBS Lett 581(27):5282-5288. https://doi.org/10. 1016/j.febslet.2007.10.022

O'Hara AM, Shanahan F (2006) The gut flora as a forgotten organ. EMBO Rep 7(7):688-693. https://doi.org/10.1038/sj.embor. 7400731

Paul K, Nonoh JO, Mikulski L, Brune A (2012) "Methanoplasmatales," Thermoplasmatales-related archaea in termite guts and other environments, are the seventh order of methanogens. Appl Environ Microbiol 78(23):8245-8253. https://doi.org/10.1128/AEM.0219312 
Pausan MR, Csorba C, Singer G, Till H, Schöpf V, Santigli E, Klug B, Högenauer C, Blohs M, Moissl-Eichinger C (2019) Exploring the archaeome: detection of archaeal signatures in the human body. Front Microbiol 10:2796-2796. https://doi.org/10.3389/fmicb. 2019.02796

Qin J, Li R, Raes J, Arumugam M, Burgdorf KS, Manichanh C, Nielsen T, Pons N, Levenez F, Yamada T (2010) A human gut microbial gene catalogue established by metagenomic sequencing. Nature 464(7285):59-65

Rak K, Rader DJ (2011) The diet-microbe morbid union. Nature 472(7341):40-41

Ramezani A, Nolin TD, Barrows IR, Serrano MG, Buck GA, Regunathan-Shenk R, West RE 3rd, Latham PS, Amdur R, Raj DS (2018) Gut colonization with methanogenic archaea lowers plasma trimethylamine $\mathrm{N}$-oxide concentrations in apolipoprotein $\mathrm{E}^{-/-}$ mice. Sci Rep 8(1):14752-14752. https://doi.org/10.1038/s41598018-33018-5

Rath S, Rud T, Pieper DH, Vital M (2020) Potential TMA-producing bacteria are ubiquitously found in mammalia. Front Microbiol 10: 2966

Roberts AB, Gu X, Buffa JA, Hurd AG, Wang Z, Zhu W, Gupta N, Skye SM, Cody DB, Levison BS (2018) Development of a gut microbetargeted nonlethal therapeutic to inhibit thrombosis potential. Nat Med 24(9):1407-1417

Sender R, Fuchs S, Milo R (2016) Revised estimates for the number of human and bacteria cells in the body. PLoS Biol 14(8):e1002533. https://doi.org/10.1371/journal.pbio.1002533

Shan Z, Sun T, Huang H, Chen S, Chen L, Luo C, Yang W, Yang X, Yao P, Cheng J (2017) Association between microbiota-dependent metabolite trimethylamine-N-oxide and type 2 diabetes. Am J Clin Nutr 106(3):888-894

Simon J-C, Marchesi JR, Mougel C, Selosse M-A (2019) Hostmicrobiota interactions: from holobiont theory to analysis. Microbiome 7(1):1-5

Soellinger A, Schwab C, Weinmaier T, Loy A, Tveit AT, Schleper C, Urich T (2016) Phylogenetic and genomic analysis of Methanomassiliicoccales in wetlands and animal intestinal tracts reveals clade-specific habitat preferences. FEMS Microbiol Ecol 92(1):fiv 149

Sogodogo E, Drancourt M, Grine G (2019) Methanogens as emerging pathogens in anaerobic abscesses. Eur J Clin Microbiol Infect Dis 38(5):811-818. https://doi.org/10.1007/s10096-019-03510-5

Sollinger A, Schwab C, Weinmaier T, Loy A, Tveit AT, Schleper C, Urich T (2016) Phylogenetic and genomic analysis of Methanomassiliicoccales in wetlands and animal intestinal tracts reveals clade-specific habitat preferences. FEMS Microbiol Ecol 92(1). https://doi.org/10.1093/femsec/fiv149

Srinivasan G, James CM, Krzycki JA (2002) Pyrrolysine encoded by UAG in Archaea: charging of a UAG-decoding specialized tRNA. Science 296(5572):1459-1462

Tang WH, Wang Z, Kennedy DJ, Wu Y, Buffa JA, Agatisa-Boyle B, Li XS, Levison BS, Hazen SL (2015) Gut microbiota-dependent trimethylamine N-oxide (TMAO) pathway contributes to both development of renal insufficiency and mortality risk in chronic kidney disease. Circ Res 116(3):448-455. https://doi.org/10.1161/ CIRCRESAHA.116.305360

Tang WW, Hazen SL (2017) Microbiome, trimethylamine N-oxide, and cardiometabolic disease. Transl Res 179:108-115

Tang WW, Wang Z, Li XS, Fan Y, Li DS, Wu Y, Hazen SL (2017) Increased trimethylamine $\mathrm{N}$-oxide portends high mortality risk independent of glycemic control in patients with type 2 diabetes mellitus. Clin Chem 63(1):297-306
Tharp JM, Ehnbom A, Liu WR (2018) tRNA(Pyl): structure, function, and applications. RNA Biol 15(4-5):441-452. https://doi.org/10. 1080/15476286.2017.1356561

Thauer RK, Kaster A-K, Seedorf H, Buckel W, Hedderich R (2008) Methanogenic archaea: ecologically relevant differences in energy conservation. Nat Rev Microbiol 6(8):579-591

Ueland PM (2011) Choline and betaine in health and disease. J Inherit Metab Dis 34(1):3-15

Vierbuchen T, Bang C, Rosigkeit H, Schmitz RA, Heine H (2017) The human-associated archaeon Methanosphaera stadtmanae is recognized through its RNA and induces TLR8-dependent NLRP3 inflammasome activation. Front Immunol 8:1535-1535. https:// doi.org/10.3389/fimmu.2017.01535

Wang Z, Klipfell E, Bennett BJ, Koeth R, Levison BS, Dugar B, Feldstein AE, Britt EB, Fu X, Chung YM, Wu Y, Schauer P, Smith JD, Allayee H, Tang WH, DiDonato JA, Lusis AJ, Hazen SL (2011) Gut flora metabolism of phosphatidylcholine promotes cardiovascular disease. Nature 472(7341):57-63. https://doi.org/10.1038/ nature09922

Wang Z, Roberts AB, Buffa JA, Levison BS, Zhu W, Org E, Gu X, Huang Y, Zamanian-Daryoush M, Culley MK (2015) Non-lethal inhibition of gut microbial trimethylamine production for the treatment of atherosclerosis. Cell 163(7):1585-1595

Warrier M, Shih DM, Burrows AC, Ferguson D, Gromovsky AD, Brown AL, Marshall S, McDaniel A, Schugar RC, Wang Z (2015) The TMAO-generating enzyme flavin monooxygenase 3 is a central regulator of cholesterol balance. Cell Rep 10(3):326-338

Willis JCW, Chin JW (2018) Mutually orthogonal pyrrolysyl-tRNA synthetase/tRNA pairs. Nat Chem 10(8):831-837. https://doi.org/ 10.1038/s41557-018-0052-5

Xu R, Wang Q, Li L (2015) A genome-wide systems analysis reveals strong link between colorectal cancer and trimethylamine $\mathrm{N}$-oxide (TMAO), a gut microbial metabolite of dietary meat and fat. BMC Genomics 16(S7):S4

Zeisel SH (1990) Choline deficiency. J Nutr Biochem 1(7):332-349

Zeisel SH, Warrier M (2017) Trimethylamine N-oxide, the microbiome, and heart and kidney disease. Annu Rev Nutr 37:157-181

Zhang Y, Baranov PV, Atkins JF, Gladyshev VN (2005) Pyrrolysine and selenocysteine use dissimilar decoding strategies. J Biol Chem 280(21):20740-20751. https://doi.org/10.1074/jbc.M501458200

Zhernakova A, Kurilshikov A, Bonder MJ, Tigchelaar EF, Schirmer M, Vatanen T, Mujagic Z, Vila AV, Falony G, Vieira-Silva S (2016) Population-based metagenomics analysis reveals markers for gut microbiome composition and diversity. Science 352(6285):565-569

Zhu W, Buffa J, Wang Z, Warrier M, Schugar R, Shih D, Gupta N, Gregory J, Org E, Fu X (2018) Flavin monooxygenase 3, the host hepatic enzyme in the metaorganismal trimethylamine N-oxidegenerating pathway, modulates platelet responsiveness and thrombosis risk. J Thromb Haemost 16(9):1857-1872

Zhu W, Gregory JC, Org E, Buffa JA, Gupta N, Wang Z, Li L, Fu X, Wu Y, Mehrabian M (2016) Gut microbial metabolite TMAO enhances platelet hyperreactivity and thrombosis risk. Cell 165(1):111-124

Zhu Y, Jameson E, Crosatti M, Schafer H, Rajakumar K, Bugg TD, Chen Y (2014) Carnitine metabolism to trimethylamine by an unusual Rieske-type oxygenase from human microbiota. Proc Natl Acad Sci U S A 111(11):4268-4273. https://doi.org/10.1073/pnas. 1316569111

Publisher's note Springer Nature remains neutral with regard to jurisdictional claims in published maps and institutional affiliations. 